\title{
An inverse optimal control problem in the electrical discharge machining
}

\author{
MARIN GOSTIMIROVIC*®), PAVEL KOVAC and MILENKO SEKULIC \\ Department of Production Engineering, Faculty of Technical Science, University of Novi Sad, Trg D. \\ Obradovica 6, Novi Sad 21000, Serbia \\ e-mail: maring@uns.ac.rs; pkovac@uns.ac.rs; milenkos@uns.ac.rs
}

MS received 4 April 2016; revised 19 February 2018; accepted 8 March 2018; published online 10 May 2018

\begin{abstract}
Electrical discharge machining (EDM) is a thermal material removal process by means of electrical discharge. Because of the stochastic nature of the EDM process, electro-thermal energy conversion in the discharge zone is still not well understood. In this paper, an inverse optimal control problem was used for analysis and optimization of energy conversion processes in order to improve machining efficiency. Modeling and identification of a thermal process were conducted using the inverse heat transfer problem based on the known temperature within a workpiece. In addition to the temperature field, this approach allows the determination of unknown heat flux density distribution on the workpiece surface. By using the heat flux, the inverse optimal control problem based on minimizing a Tikhonov functional allows to obtain the optimal heat source parameters (discharge power and discharge duration) on the discharge energy. In this context, the concept of inverse problem allows reliable determination of the optimal discharge energy to achieve the highest possible productivity with the desired quality. The performance of prediction of the heat affected zone compared to the experimental results showed a good agreement, which confirms the validity of the inverse method compared to the reported models.
\end{abstract}

Keywords. EDM process; discharge energy; heat source parameters; inverse problem; optimization.

\section{Introduction}

Electrical discharge machining (EDM) is basically a complex process in which the mechanism of material removal is based on transformation of electrical energy into thermal energy. Non-contact energy conversion between the electrodes (tool and workpiece) immersed in a dielectric fluid, results in local melting and vaporization of the workpiece material. EDM is a nontraditional process capable of machining electrically conductive materials regardless of their physical and mechanical properties. It is primarily used to produce high quality parts with complicated geometry that cannot be achieved by conventional machining processes. In this context, there can be little doubt that EDM process shall remain important in modern manufacturing engineering [1-3].

Despite its distinguished importance, EDM is a process that has certain limitations with respect to the material removal due to the production of large quantities of heat during the electro-thermal energy conversion. In energy conversion a plasma zone is formed in the small volume at the shortest distance between the electrodes and very quickly reaches an extremely high temperature range over

*For correspondence $10,000^{\circ} \mathrm{C}$ [4]. In this context, periodic discharge processes lead to high material removal rate, but also the certain surface roughness, heat affected zone, tool wear, etc. [5, 6].

Most attempts to explain the nature and principles of material removal in EDM process reported in the literature have been based on theoretical concepts of thermal-physics. The first analysis included one-dimensional analytical models. These models have been investigated by a number of researchers since 1970 [7-9]. More elaborate approaches utilizing different two-dimensional heat transfer models then followed [10, 11]. In the most recent attempts, researchers used three-dimensional numerical methods for determining the thermal state of the EDM discharge zone $[12,13]$.

Despite the availability of the aforementioned studies, impact of the discharge thermal on the machining performance of EDM process is fairly complex and not completely solved yet. The main obstacle lies in the difficulty in determination and monitoring of EDM discharge temperature. Systematic research in EDM has yielded a number of various analytical and experimental methods for determination of temperatures in the narrow and wider discharge zone. Due to the complexity of the measuring technique the first studies on temperatures in EDM were mainly theoretical $[4,14]$. On the other hand, only the latest 
advancements in measuring equipment allow development of the experimental methods for temperature measurement in EDM. A most number of researches have been done on measurement of the temperature distribution of the EDM based on the optical emission spectroscopy and resistancemeasuring system [15-17].

Based on the previously mentioned works, although thermal properties of the EDM process are very important, this research is fairly sophisticated. A review of the recent research shows that non-linear and non-stationary processes, involving intense heat transfer such as the EDM, can be successfully solved using approaches based on the solution of inverse problems of heat transfer.

In the case of thermal processes in machining, inverse problems are most often applied for identification, modeling and optimization. The inverse heat transfer problem represents a good alternative to evaluation of the behavior of the cutting temperature from the machining conditions [18]. Inverse problem solving technique has been frequently used for studying the distribution of the heat flux on tool-workpiece contact area [19]. A number of researchers are using the inverse method as a way for optimization of the thermal aspects in machining [20]. In all previous works, inverse approach has been applied in the conventional machining processes. Inverse problem is much less used in the EDM. This method so far has been used to identify EDM process by means of approximation of the temperature in discharge zone [21-23].

This paper takes a new approach to study the discharge energy on the machining characteristics in electrical discharge machining, using an inverse optimal control problem. The inverse optimal control problem was used for the first time to modeling and optimization of the thermal performance that leads to an allowed heat flux in the surface layer of the workpiece in the given EDM conditions. In contrast to previous research, this approach of using the heat flux enables the optimal control relation of the heat source power and its time duration of a single pulse discharge. In this respect, thermal model based on inverse heat transfer problem has been developed to determine the heat flux density in EDM process. Further, inverse problem in which heat flux distribution is calculated for assumed boundary conditions, can be used to estimate the workpiece temperature. Knowing the heat flux distribution could also be accomplished an optimal control method of the discharge energy of a single pulse. In this context, the main aim in using the inverse method was to predict the thermal performances of the EDM process with determination of the optimal machining parameters. The performance of prediction model is compared to the experimental results and good agreement was found between them.

\section{Inverse and optimization problems in EDM process}

Inverse heat transfer problem represents an innovative paradigm in research and development of thermal processes and systems involving intense heat exchange. Inverse problem allows the closest possible mathematical approximation of the real heat transfer based on limited experiments. In this context, inverse problems have become the most important practical applications for different thermal analysis [24-26].

In the research of the thermal processes, if there exists complete description of the mathematical model (domain, properties, boundary and initial conditions), this is usually referred to as a direct problem of heat transfer. If any of the conditions necessary to define a direct problem are unknown, it is called an inverse problem. The inverse heat transfer problems have been generally associated with the estimation of an unknown temperature or heat flux boundary condition. However, how to get a solution of inverse problem, additional conditions must be provided, e.g., temperature inside a domain. Therefore, inverse heat transfer problems are mathematically classified as ill-posed, i.e., these problems are characterized by the non-uniqueness and instability of solution [27, 28].

The inverse optimal control problem has been employed to overcome the ill-posed nature of the inverse heat transfer problem. Despite their similarities, inverse problem and inverse optimal control problem are conceptually different. The inverse problem is concerned with the modeling and identification of thermal process. On the other hand, the inverse optimal control problem generally deals with the minimization or maximization of a certain objective quality function, in order to find variables that will result in desired results. Inverse optimal control problems are much more difficult than standard inverse problems. There are numerous optimization techniques that could be employed in this problem. Most of the literature [20, 25] uses a robust gradient-based optimization method for the target localization. This method is one of the most effective nonlinear optimization algorithms to guarantee the quality of inverse optimal control problem solution and measurements.

In contrast to inverse problem, uniqueness and stability of the solution do not need to be an important issue for inverse optimal control problem as long as the solution can be practically implemented. However, sometimes the decision is more subjective, because different criterion can be idealized as a more sustainable solution.

\subsection{Energy partition in EDM process}

EDM is the thermal process in which material is removed by a series of recurring electrical discharges between the tool and workpiece electrodes. One of the essential problems of EDM process is knowledge of thermal energy distribution, namely, character of the temperature field within discharge zone. Thermal load of discharge during the EDM process can be defined by the heat source and sink characteristics. Transformation of electrical energy acts as a heat source and has recurring character. Tool, workpiece and dielectric act as heat sinks and they allow evacuating heat by conduction, convection and radiation. 
The effects of EDM heat source/sink could be judged by the type, dimensions, shape, power and duration [7]. Considering the fact that the heat is generated and distributed in a relatively closed space between the electrodes, it is classified as an internal heat flow. Heat flow is small and exactly defined area. Heat flow has fixed location and his effect is immediate, but is extremely intense.

The power of the heat source in the discharge zone is expressed by means of heat flux density, and it is given as:

$$
q_{e}=\frac{Q}{\int d S \int d t}
$$

where $Q$ is the heat energy and $S$ is the surface area.

EDM process considers the fact that the heat energy equivalent to discharge energy [29], namely:

$$
Q=E_{e} \cong U_{e} \cdot I_{e} \cdot t_{e}
$$

where $U_{e}$ is the discharge voltage, $I_{e}$ is the discharge current and $t_{e}$ is the discharge duration.

By using Eqs. (1) and (2), there follows an expression for discharge power of a single pulse:

$$
q_{e} \cong \frac{U_{e} \cdot I_{e}}{r_{e}^{2} \pi}
$$

where $r_{e}$ is the radius of the heat source in the discharge zone.

The duration of the heat source is identical to the discharge duration, and is considered equal to pulse duration:

$$
t_{e} \cong t_{i}
$$

Heat flow characteristics depend on the EDM conditions as well as the chemical-physical material properties of the workpiece, tool and dielectric fluid. Considering the efficiency of EDM, the heat quantity evacuated through the workpiece is of most importance. Most researchers observed that small fraction of total discharge energy is evacuated as heat into the workpiece, and the rest is lost into the tool and dielectric fluid $[4,9]$.

Based on the aforementioned, assessment of the heat distribution problem is one of the crucial investigation problems during EDM process. The amount of the total heat which reaches to the workpiece is determined by the energy partition which can be defined with the following equation:

$$
R_{q}=\frac{q_{w}}{q_{e}}
$$

where $q_{w}$ is the heat flux to the workpiece.

In the heat transfer theory, the Green's function method for a point source is generally accepted as the fundamental for analytical determination of the energy partition [30]. If assumed that ideally isolated source of heat $q$ is used, which has a specific thermal-physical characteristics $k \rho c$ ( $k$ thermal conductivity, $\rho$-material density, $c$-specific heat capacity), in case of maximum temperature there follows the final expression:

$$
T_{\max }=\frac{2}{\sqrt{\pi}} \cdot q \sqrt{\frac{t}{(k \rho c)}}
$$

The energy partition is determined starting from the fact that the fraction of discharge heat goes to the workpiece $q_{w}=R_{q} q_{e}$ and rest to the tool and dielectric fluid $\left(1-R_{q}\right) q_{e}$. In that case, for equal temperatures on the surface of workpiece and the discharge zone, using the Eq. (5), there follows an equation for the energy partition:

$$
R_{q}=\frac{1}{1+\sqrt{\frac{(k \rho c)_{c}}{(k \rho c)_{w}}}}
$$

Here is $(k \rho c)_{w}$ the thermal-physical properties of workpiece material and $(k \rho c)_{c}$ is the thermal-physical properties of the composite which involves tool material and dielectric fluid, which can be determined by following equations:

$$
\begin{gathered}
k_{c}=\phi k_{t}+(1-\phi) k_{d} \\
(\rho c)_{c}=\phi(\rho c)_{t}+(1-\phi)(\rho c)_{d}
\end{gathered}
$$

where $\phi$ is the empirically determined constant and represents the fraction of thermal-physical properties of the tool/ dielectric composite material.

\subsection{Inverse modeling of the EDM process}

The first step in the inverse research of any thermal process is development of a model which is valid for a limited field with given boundary conditions [24, 25]. The model, which adequately describes the real process, allows to correlate input and output parameters which define the state of the process at any time. However, it is well known that it is very difficult to modeling of the thermal phenomena in the EDM. If all variable parameters were taken into consideration, thermal modeling of the EDM process would become an impossible task. Therefore, some simplifications are necessary for any model, thus, and for the inverse problem in $\operatorname{EDM}[19,22]$.

In order to mathematically formulate the inverse heat transfer problem in EDM process, the relatively simple thermal model to simulate a single electrical discharge is used (figure 1). In electrical discharge machining, the heat source in the discharge zone can be treated with uniform heat distribution [7,9]. The heat of the discharge zone transfers only by conduction. The above assumptions are a valid approximation in case of the heating of a small volume of workpiece material. Furthermore, if we disregard the dissipation of heat flow, then the workpiece can be approximated as a semi-infinite cylinder.

As previously mentioned, the inverse problem is used to assess some input characteristics. To identify and control 


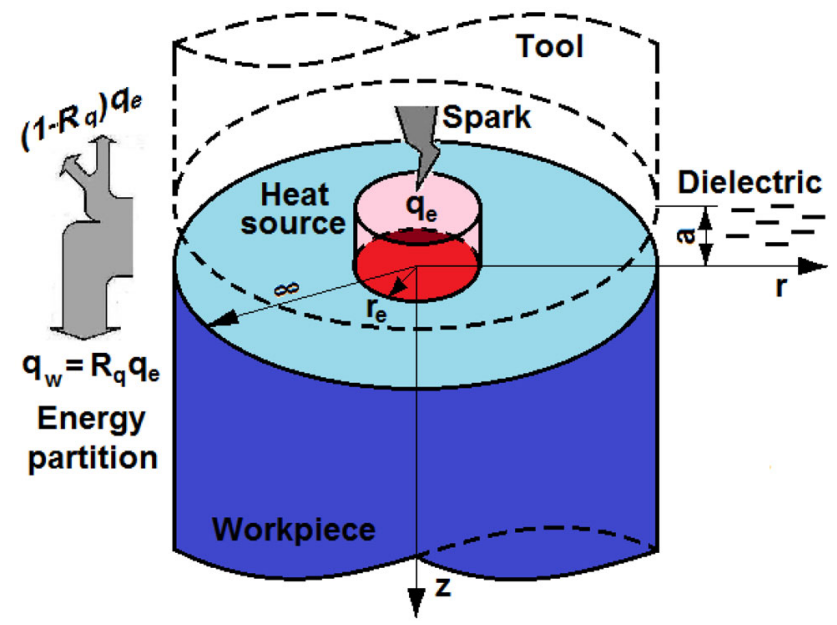

Figure 1. Thermal model of EDM.

the thermal state of the EDM process, one needs to determine the boundary condition: heat flux density in the discharge zone $q_{e}$. In that case, for defined thermal model of EDM the following is the simplest type of differential equation of heat conduction in two-dimensional cylindrical coordinate system:

$$
\rho c \frac{\partial T}{\partial t}=k\left(\frac{1}{r} \frac{\partial T}{\partial r}+\frac{\partial^{2} T}{\partial r^{2}}+\frac{\partial^{2} T}{\partial z^{2}}\right)
$$

where $T$ is the temperature, $r$ is the radial cylindrical coordinate, $z$ is the axial cylindrical coordinate, $t$ is the time, $k$ is the thermal conductivity, $\rho$ is the material density and $c$ is the specific heat.

In order to formulate the solution of differential Eq. (10) by using the inverse problem, there must be introduced an initial, boundary and additional conditions.

The initial condition refers to defining a temperature distribution in the discharge zone at the initial moment $t=0 \mathrm{~s}$, and can be taken as room temperature of the dielectric fluid in which the electrodes are dipped:

$$
\left.T(r, z, t)\right|_{t=0}=T_{0}
$$

Known boundaries of the considered domain are supposed to be defined with a cylindrical workpiece by the boundary condition of the second order:

$$
\begin{aligned}
& -\left.k \frac{\partial T(r, z, t)}{\partial z}\right|_{z=\infty}=0 \\
& -\left.k \frac{\partial T(r, z, t)}{\partial r}\right|_{r=\infty}=0
\end{aligned}
$$

An additional condition is represented as the known constant temperature at a certain point on the workpiece $z=H(0<H \leq \infty)$. Thereby, the additional condition is derived if it is assumed that inside workpiece temperature was not allowed to exceed the critical temperature heat affected zone (HAZ).

$$
\left.T(r, z, t)\right|_{\substack{z=H \\ r=0}}=T_{c}
$$

The ultimate solution of the inverse heat transfer problem is to determine the unknown boundary condition, namely heat flux to the workpiece $q_{w}(t)=R_{q} q_{e}(t)$, as well as the entire temperature distribution $T=T(r, \mathrm{z}, t)$, through the elementary section of the workpiece, $\boldsymbol{D}=\{(r, z, t)$ : $\left.r \in[0, \infty], z \in[0, \infty], t \in\left[0, t_{e}\right]\right\}$.

$$
-\left.k \frac{\partial T(r, z, t)}{\partial z}\right|_{\substack{z=0 \\ r<r_{e}}}=q_{w}(t)
$$

To solve the inverse heat transfer problem requires the initial task (Eqs. (10) to (13)) to be divided into two separate problems: the inverse problem $\boldsymbol{D}_{1}$ and the direct problem $\boldsymbol{D}_{2}$ (figure 2).

First, a direct problem separated as a portion of the body can be analyzed as a well-posed problem because there are known boundary conditions within the area $\boldsymbol{D}_{2}=\{(r, z, t)$ : $\left.r \in[0, \infty], z \in[\mathrm{H}, \infty], t \in\left[0, t_{e}\right]\right\}$. From this, heat flux at a certain point $q_{H}(t)$ can be found from the solution for the temperature distribution $T=T(r, \mathrm{z}, t)$ within the area $\boldsymbol{D}_{2}$.

$$
-\left.k \frac{\partial T(r, z, t)}{\partial z}\right|_{\substack{z=H \\ r=0}}=q_{H}(t)
$$

The density heat flux $q_{H}(t)$ that has been previously determined, can be connected to the area $\boldsymbol{D}_{1}=\{(r, z, t)$ : $\left.r \in[0, \infty], z \in[0, H], t \in\left[0, t_{e}\right]\right\}$. Consequently, two conditions are specified at $z=H$ and neither one at $z=0$. Such a set of boundary conditions is related to an ill-posed mathematical problem. In this context, inverse problem is solved starting from the known boundary conditions at one border. Solved represents the temperature distribution $T=T(r, \mathrm{z}, t)$ within the area $\boldsymbol{D}_{1}$ and unknown heat flux boundary condition on the workpiece surface $q_{w}(t)$.

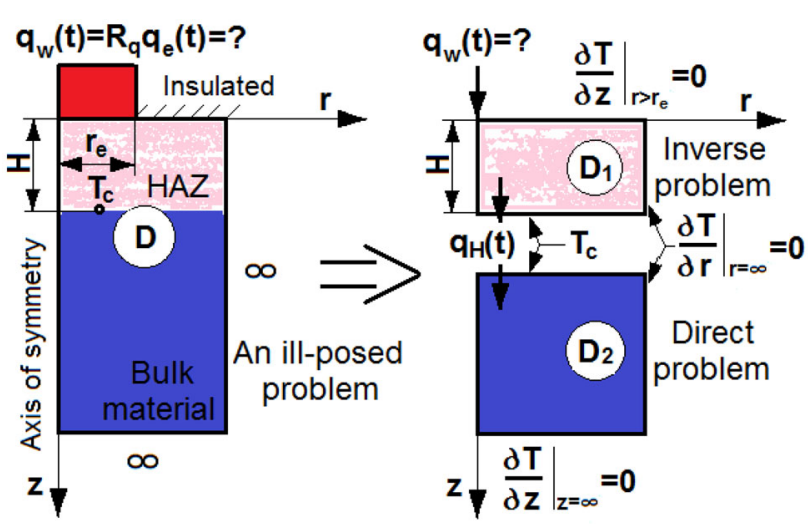

Figure 2. Inverse heat transfer problem in EDM process. 
For solving the partial differential equations which describe the process of heat conduction are mostly used approximate numerical methods. The finite element method (FEM) is a popular numerical approach used for solving problems in different areas of engineering. The FEM is based on variational techniques, where is possible to replace the problem of integrating a differential equation by the equivalent function that gives a minimum value of integral [31].

There are more methods that are applied to solving problems using the FEM method. Galerkin's method is one of the popular choices. The finite element method that is used to solve partial differential equations of heat conduction (Eq. (10)) using the Galerkin's method it can be expressed in matrix form as:

$$
[k]\{T\}+[c]\left\{\frac{\partial T}{\partial t}\right\}=\{q\}
$$

where $[k]$ is the thermal conductivity matrix; $[c]$ is the specific heat matrix; $\{T\}$ is the temperature vector and $\{q\}$ is the heat flux vector.

By applying the central difference method in time domain to the Eq. (16), the following equation is obtained:

$$
\begin{aligned}
\left([c]+\frac{\Delta t}{2}[k]\right)\{T\}_{t+\Delta t} & =\left([c]+\frac{\Delta t}{2}[k]\right)\{T\}_{t} \\
& +\frac{\Delta t}{2}\left(\{q\}_{t+\Delta t}+\{q\}_{t}\right)
\end{aligned}
$$

The Eq. (17) is a system of linear simultaneous equations that is solved by the computer for the nodal temperatures at time $t+\Delta t$, where $\Delta t$ is the time discretization.

\subsection{Inverse optimal control approach}

One of the perspective ways to solve inverse heat transfer problem involves its transformation as an approximate well-posed problem using some kind of optimal strategy. Optimal control theory defines how solutions may be found for such ill-posed problems. One method for determining these solutions are related to approach via quadratic functional based on mean-square norms, wherein the ill-posed problem is solved as a constrained minimization of a chosen functional. In this context, the inverse optimal control problem for the selected quality optimization algorithm, allows in obtaining the optimal thermal conditions by using control function.

In electrical discharge machining, optimization of thermal process is to determine the state and control function, namely the temperature distribution $T=T(r, \mathrm{z}, t)$ and the heat flux density $q_{w}(t)$, so as to satisfy the mathematical form of the inverse heat transfer problem, as defined by equations (10) to (14). These functions are determined with the additional condition when the known temperature at a certain point on the workpiece $T_{c}=T(0, H, t)$ is consistent with the temperature $T_{q}=T(0, H, t)$ which is calculated based on control function of the process $q_{w}(t)$ by the Tikhonov functional [27]:

$$
J(q)=\int_{0}^{t_{e}}\left[T_{q}(0, H, t)-T_{c}(0, H, t)\right]^{2} d t
$$

In general, many optimal control techniques may determine a solution that minimizes or maximizes a certain objective quality function. There are numerous optimization techniques, but in most cases an iterative optimization method is needed. In this case, an iterative method of optimization that uses feedback loops in order to optimize the response of interest is used. The inverse problem of optimization which uses an iterative method supposes that it is the unknown solution of the problem that is minimizing the quality function.

For practical application, the integral control function Eq. (18) can be approximated by the finite sum:

$$
J(q) \cong \Delta t \sum_{n=1}^{e}\left[T_{q}\left(0, H, t_{n}\right)-T_{c}\left(0, H, t_{n}\right)\right]^{2}
$$

Effective ways to optimization of objective function using minimization of the differences between the known and the calculated temperature is presented as an iterative method of optimization.

Establishing the inverse optimal control problem, through gradual approach to the optimum level over a number of successive steps, gives exact solution using the following form:

$$
q_{i+1}(t)=q_{i}(t)+\Delta q_{i}(t) ; \quad i=0,1,2, \ldots
$$

where $q_{0}(t)$ is the initial approximation and $\Delta q_{i}(t)$ is the iteration step.

By applying the optimization technique, the Tikhonov functional $J\left(q_{i}\right)$ is calculated for particular values of density heat flux $q_{i}(t)$. The iterative procedure is repeated until the minimum value of the functional $J\left(q_{i+1}\right)<J\left(q_{i}\right)$ is reached. The iterative method procedure is considered finished for a sufficiently small functional, which means that the calculated and the known temperatures are very close and may be considered identical:

$$
\min _{q} J(q)
$$

\section{Results and analysis}

\subsection{Experiments}

The experiments were conducted on a CNC industrial diesinking EDM machine tool, manufactured by Fanuc. The main specifications of the pulse generator are: range of 
discharge current is $I_{e}=0-100 \mathrm{~A}$, range of pulse duration is $t_{i}=0-1000 \mu \mathrm{s}$, range of pulse off time is $t_{o}=0-$ $100 \mu \mathrm{s}$ and range of open gap voltage is $U_{o}=0-100 \mathrm{~V}$. The dielectric was petroleum $\left(830 \mathrm{~kg} / \mathrm{m}^{3}\right.$ density, $3.1 \mathrm{~W} /$ $\mathrm{m}{ }^{\circ} \mathrm{C}$ thermal conductivity, $680 \mathrm{~J} / \mathrm{kg}{ }^{\circ} \mathrm{C}$ specific heat, $126^{\circ} \mathrm{C}$ ignition temperature, and $5.3 \mathrm{cSt}$ viscosity) with the natural flushing.

The workpiece material used in the experiments was manganese alloyed cold-work tool steel, AISI O2 $(0.9 \% \mathrm{C}$, $2 \% \mathrm{Mn}, 0.3 \% \mathrm{Cr}$, and $0.2 \% \mathrm{~V}$ chemical properties) with a hardness of 62 HRC. The tool was made of $99.9 \%$ pure electrolytic copper with a cross-section dimension of $20 \times 10 \mathrm{~mm}$. The major physical properties of the workpiece and tool materials used in this study are given in table 1 .

The machining conditions setting system includes the discharge current and the pulse duration, and the discharge energy (Eq. 2). The discharge current and pulse duration was chosen to achieve the maximum material removal rate and minimum tool wear. The rest of the parameters of electrical pulse were held constant, according to manufacturer's recommendations (the open gap voltage is $U_{o}=100 \mathrm{~V}$, the duty factor is $\tau=0.8$ and the positive tool electrode polarity).

In this study, measuring material removal rate (MRR) was the first priority of machining performance. The material removal rate was measured by monitoring the total machining time for a certain depth of eroding material using the machine's CNC control system. Metallographic examination of the heat affected zone (HAZ) was conducted on more specimens. Metallographic examinations were performed on an optical microscope, model ARISTOMET, manufactured by Leit, Germany.

Figure 3 shows the results of experimental investigation under specific machining conditions. The diagram shows the dependence of material removal rate on the pulse duration for different discharge currents. The experimental results show that for every discharge current $I_{e}$ there is a corresponding optimal pulse duration $t_{i(o p t)}$ which specifies the maximum material removal rate $M R R_{\text {max }}$. Its value increases with the increase of the discharge current. On the other side, at constant pulse duration an increase of the discharge current increases the material removal rate. This

Table 1. Properties of the electrode materials.

\begin{tabular}{lcc}
\hline Materials & AISI O2 & Copper \\
\hline Density $\left(\mathrm{kg} / \mathrm{m}^{3}\right)$ & 7750 & 8960 \\
Thermal conductivity $\left(\mathrm{W} / \mathrm{m}{ }^{\circ} \mathrm{C}\right)$ & 15.1 & 401 \\
Specific heat $\left(\mathrm{J} / \mathrm{kg}{ }^{\circ} \mathrm{C}\right)$ & 480 & 385 \\
Modulus of elasticity $(\mathrm{GPa})$ & 193 & 110 \\
Melting point $\left({ }^{\circ} \mathrm{C}\right)$ & 1450 & 1083 \\
Electrical resistivity $(\mu \Omega \mathrm{cm})$ & 72 & 1.69 \\
Poisson's ratio & 0.31 & 0.34 \\
\hline
\end{tabular}

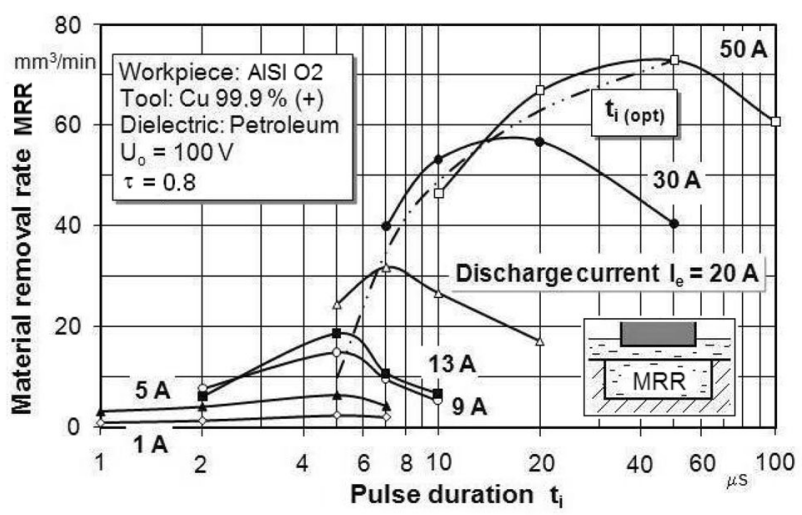

Figure 3. Influence of EDM parameters on MRR.

efficiently precludes us from unambiguous determination of the influence of electrical pulse parameters on the material removal rate.

Metallographic examinations showed the existence of a melted and resolidified layer and heat affected zone in all cases of process execution. The heat affected zone is characterized by microstructure and microhardness changes compared to the bulk material. Metallographic picture of the sample reveals two characteristic layers: hardened layer and tempered layer. Thereby, the hardened layer has higher microhardness, while the tempered layer has lower microhardness. Higher microhardness is the result of the austenitic-martensitic phase transition, while the lower microhardness occurs due to formation of a mixture of martensite and cementite. That means that the surface temperature of the workpiece exceeded the temperature of tempering, which equals $520^{\circ} \mathrm{C}$ for the selected tool steel. An example of microstructure of the heat affected zone (HAZ) in the surface layer of the workpiece material for the middle discharge energy is shown in figure 4.

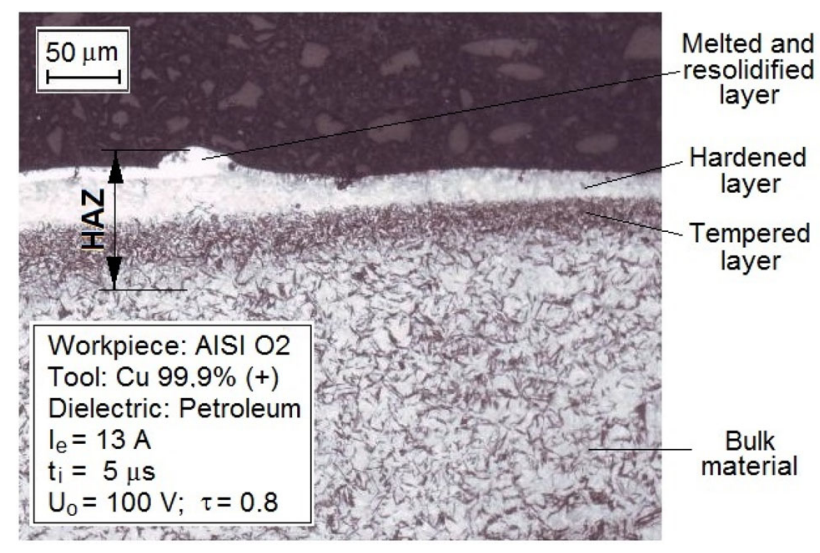

Figure 4. Microstructure of heat affected zone in EDM. 


\subsection{The results of the inverse modeling}

The inverse modeling of the EDM process is performed with the use of numerical methods (section 2.2) and experimental results (section 3.1). In the model, the outer radius of the insulated surface of the electrodes is estimated on the basis of the previous studies as 50 radius of the heat source $[7,11]$. The experimentally measured value of gap distance was used to model EDM [29]. In addition, the important step is to introduce the physical properties of the electrode materials (table 1) and the energy partition (Eq. 7). Software ANSYS R16.1 is used to create an axisymmetric finite element model of the EDM process (figure 5).

In case of verification the EDM process by means of inverse heat transfer problem, following input parameters were taken. The initial condition is at ambient temperature of the dielectric fluid of $T_{0}=20^{\circ} \mathrm{C}$. The boundary condition is heat flux on the lower boundary of the workpiece surface $q_{\infty}=0 \mathrm{~W} / \mathrm{m}^{2}$. The additional condition is the known critical tempering temperature $\left(T_{c}=520^{\circ} \mathrm{C}\right.$ for the selected steel) inside the workpiece at the depth of material which was without heat affected zone during the EDM process $(H=H A Z=80 \mu \mathrm{m}$ for the selected example, figure 4).

Based on the previously presented assumptions, the temperature distribution in the discharge zone was obtained by computation of a single pulse. The temperature distribution in the electrodes is computed by inverse heat transfer problem. Using ANSYS finite element code, threedimensional temperature field model in EDM process is shown in figure 5 . The figure illustrates the total temperature field of the electrodes surface layer for one machining condition $\quad\left(I_{e}=13 \mathrm{~A}, \quad t_{i}=5 \mu \mathrm{s}, \quad a=0.155 \mathrm{~mm}\right.$, $R_{q}=26 \%$ ). Under these conditions, the highest temperature in the workpiece was found to be $3608^{\circ} \mathrm{C}$.

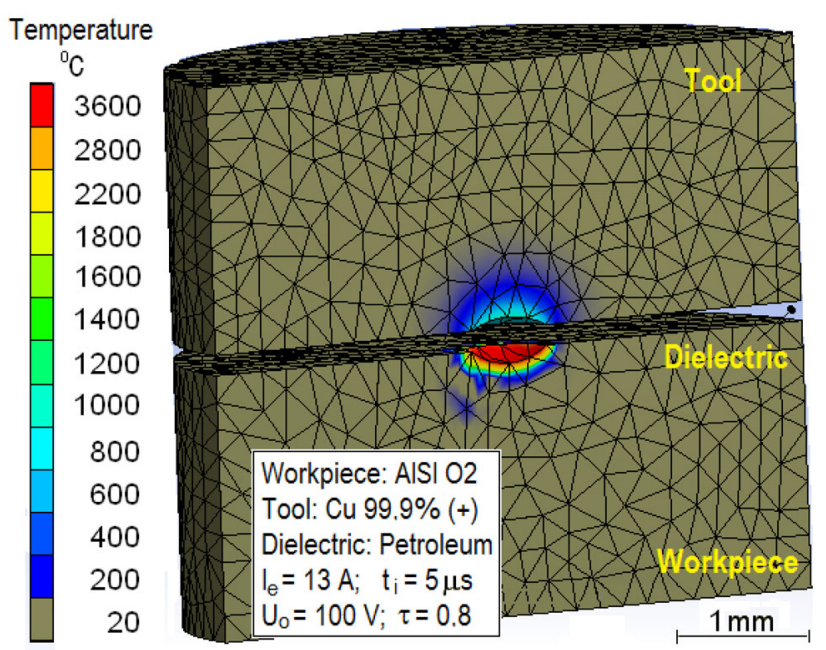

Figure 5. Inverse FEM model of temperature distribution at the end of single pulse.
With a temperature distribution, the inverse heat transfer problem is concerned with the determination of the unknown boundary condition, namely heat flux to the workpiece $q_{w}(t)$. Radius of the heat source is equivalent to the plasma channel and for this case it is determined from the isotherm profile as $50 \%$ from the material removed from the superheated area (figure 5). A three-dimensional finite element heat flux model and change of the heat flux density during discharge duration of a single EDM pulse are shown in figure 6 . The calculation of the distribution of heat flux density defines heat load on the workpiece and shows the direct relationship between heat source parameters, i.e., discharge power and its time duration.

\subsection{Procedure of the inverse optimal solution}

As previously mentioned, effectiveness of EDM mostly depends on the discharge energy and situation of the heat source parameters of a single pulse. Thereby, the heat source duration increases with discharge energy, but the heat source power has decreasing trend. This might be due to the fact that higher discharge energy increases the radius of the heat source, and thereby lowers heat flux density to the workpiece. Consequently, the basic process parameters of electrical discharge machining have the reverse effect on the change of parameters of the heat source, which largely complicates optimal control of EDM.

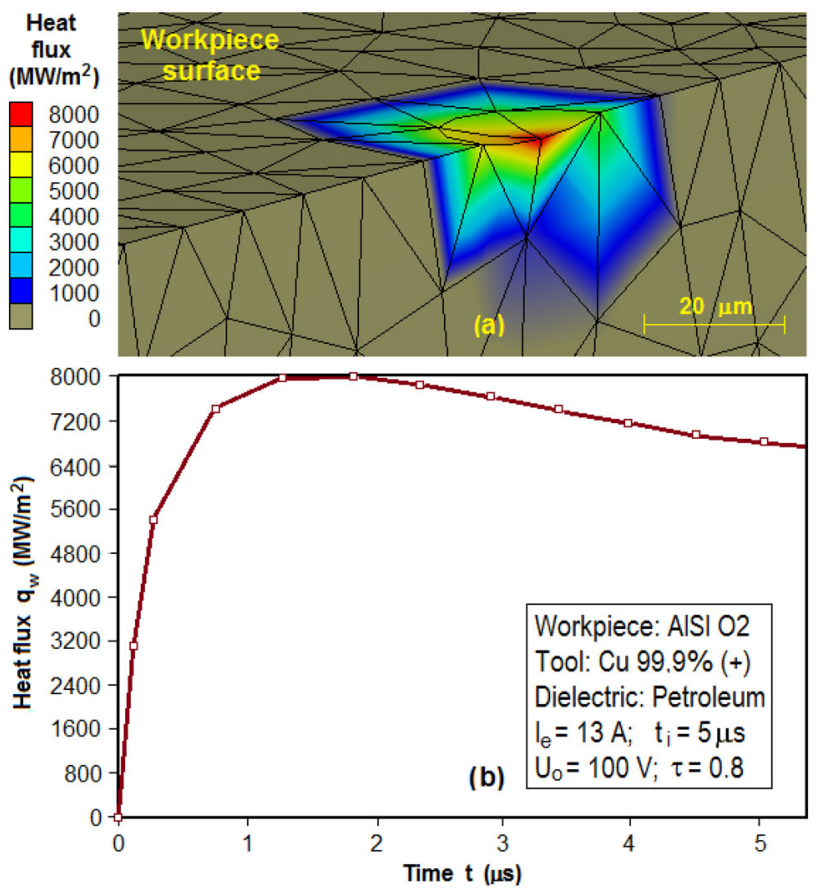

Figure 6. Inverse estimation of the heat flux distribution to the workpiece surface: (a) 3D heat flux field, (b) the heat flux during discharge duration. 
For these reasons, this paper focuses on the optimization of EDM machining parameters such that the heat load on the workpiece does not exceed the limits allowed by the specification. Based on the previously defined input parameters and additional conditions, the optimal relationship between heat flux parameters is calculated and subsequently used to find optimal EDM machining parameters (figure 7). For the purpose of calculating the mentioned values of heat flux parameters, an iterative method of optimization was used, written on the basis of earlier proposed algorithm by the authors [20]. The process of optimization is conducted through gradual approach to the optimum level, based on the minimization of the Tikhonov functional. In this context, the inverse optimal control problem provides a method for easy and precise selection of EDM input parameters. In this case, the optimal control of EDM process is reduced to the optimization of two machining parameters: discharge current and pulse duration. Optimal discharge current and pulse duration can be inside or on the very boundary of the search domain. In this case, based on the previously defined machining conditions, for the selected steel was found to be the optimal discharge current $I_{e}=5 \mathrm{~A}$ and pulse duration $t_{i}=2 \mu \mathrm{s}$.

In order to completely verify the good behavior of the model and the numerical solution procedure of inverse optimal control problems in the electrical discharge machining, testing was performed with a wide variation of input data. In this model, discharge current and pulse duration are the input variables and output is the thickness of the heat affected zone in the EDM process (figure 8). The proposed inverse optimal control model provides the precise selection of the input machining parameters on the maximum productivity with the minimum endangerment of functional properties of the finished product.

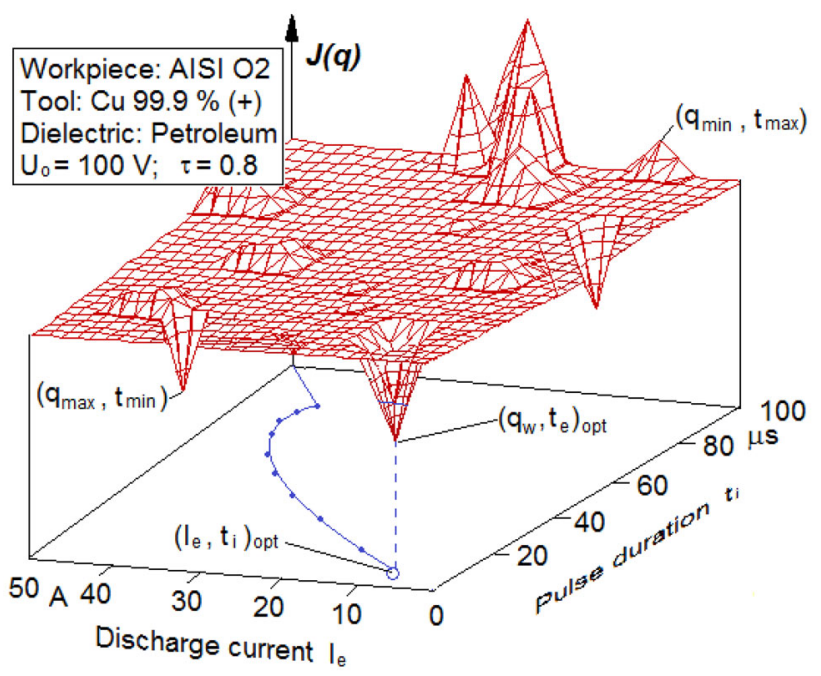

Figure 7. Iterative optimization method for locating the most favorable heat flux parameters.

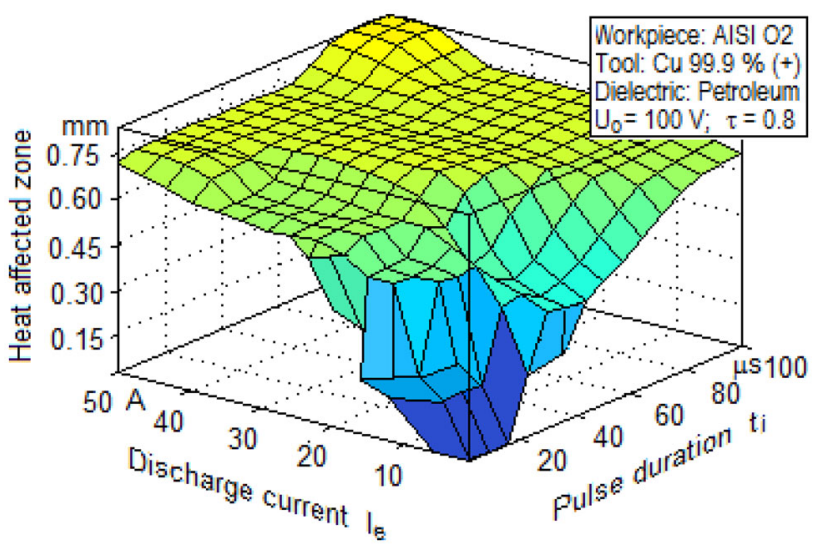

Figure 8. Inverse modeling of the heat affected zone in EDM process.

\subsection{Discussions}

Based on the previously conducted experimental-mathematical research it was established that the performance and efficiency of the EDM directly depends on the generation and distribution of the discharge energy, i.e., the heat source parameters (discharge power and discharge duration). Thereby, the increase of the discharge energy, either through discharge power or duration, increases the material removal rate, but also the existence of the heat affected zone. In this context, there exists an optimal discharge energy which yields the best possible results in machining.

In practice, the discharge energy can be changed with two EDM machining parameters: discharge current and pulse duration. However, these two parameters have the opposite effect on the change of the discharge energy and the optimization is conducted using the inverse optimal control problem approach.

Inverse control of EDM discharge energy is investigated through experimental verification. The comparison between the computed and the experimental results showed that the inverse optimal control problem is capable of producing reliable data. The differences can be explained by a number of factors which had to be omitted or estimated in the course of the numerical modeling of the effect of inputoutput parameters in EDM process. It can thus be concluded that inverse modeling will give better monitoring and optimization of EDM performance compared to the reported models.

Judging by the above, the inverse optimal control problem has yielded encouraging modeling results and has proven to be industrially feasible in the case of optimization of electrical discharge machining process parameters. Coupled with the appropriate database, the inverse optimization shows good industrial applicability inthe reduction of heat affected zone in EDM process. One possible application is in the machining of the difficult-to-machine materials and complex geometry parts. 


\section{Conclusions}

The inverse heat transfer problem in EDM process is a practical way to reliably investigate the generation and distribution of thermal energy in the discharge zone, using an electro-thermal model. Values predicted by inverse model agree with the experimental results. In this respect, the inverse heat transfer problem shows good directions which could be followed in future researches into the electrical discharge machining.

The Inverse modeling of EDM allows determination of the temperature distribution reached in the discharge zone and heat flux density on the workpiece surface at the end of each heat transfer cycle. The stability of the inverse numerical solution largely depends not only on the additional condition, i.e., the known temperature inside the workpiece, but also the thermal-physical values of the electrode materials.

The inverse optimal control problem is based on the locating the most favorable heat flux parameters of the EDM process. Inverse problem regulates the discharge power and the discharge duration to be kept within limits which allows reliable determination of the optimal machining parameters.

\section{Acknowledgements}

The paper is the result of the research within the project TR 35015 financed by the Ministry of Education, Science and Technological Development of the Republic of Serbia.

\section{List of symbols}

a Gap distance, $\mathrm{m}$

c Specific heat, $\mathrm{J} / \mathrm{kg}{ }^{\circ} \mathrm{C}$

$E_{e} \quad$ Discharge energy, $\mathrm{J}$

$I_{e} \quad$ Discharge current, A

$J \quad$ Tikhonov functional

$H \quad$ Boundary layer thickness, $\mathrm{m}$

$H A Z$ Heat affected zone, $\mathrm{m}$

$k \quad$ Thermal conductivity, W/m ${ }^{\circ} \mathrm{C}$

$Q \quad$ Heat energy, J

$q \quad$ Heat flux density, $\mathrm{W} / \mathrm{m}^{2}$

$q_{e} \quad$ Discharge heat, $\mathrm{W} / \mathrm{m}^{2}$

$q_{H} \quad$ Heat flux at a certain point, $\mathrm{W} / \mathrm{m}^{2}$

$q_{w} \quad$ Heat flux to the workpiece, $\mathrm{W} / \mathrm{m}^{2}$

$r \quad$ Radial cylindrical coordinate, $\mathrm{m}$

$r_{e} \quad$ Radius of heat source, $\mathrm{m}$

$R_{q} \quad$ Energy partition

$t \quad$ Time, s

$t_{e} \quad$ Discharge duration, $\mathrm{s}$

$t_{i} \quad$ Pulse duration, $\mathrm{s}$

$T \quad$ Temperature, ${ }^{\circ} \mathrm{C}$

$T_{c} \quad$ Known critical temperature, ${ }^{\circ} \mathrm{C}$

$T_{0} \quad$ Initial temperature, ${ }^{\circ} \mathrm{C}$
$T_{q} \quad$ Calculated temperature, ${ }^{\circ} \mathrm{C}$

$U_{e} \quad$ Discharge voltage, $\mathrm{V}$

$U_{o} \quad$ Open gap voltage, $\mathrm{V}$

$M R R$ Material removal rate, $\mathrm{mm}^{3} / \mathrm{min}$

$z \quad$ Axial cylindrical coordinate, $\mathrm{m}$

$\rho \quad$ Material density, $\mathrm{kg} / \mathrm{m}^{3}$

$\tau \quad$ Duty factor

\section{References}

[1] König W, Wertheim R, Zvirin Y and Roren M 1975 Material removal and energy distribution in electrical discharge machining. CIRP Ann. 24: 95-100

[2] Heckele M and Schomburg W K 2004 Review on micro molding of thermoplastic polymers. J. Micromech. Microeng. 14: 1-14

[3] Padhee S, Nayak N, Panda S K, Dhal P R and Mahapatra S S 2012 Multi-objective parametric optimization of powder mixed electro-discharge machining using response surface methodology and non-dominated sorting genetic algorithm. Sadhana-Acad. Proc. Eng. Sci. 31: 223-240

[4] Yeo S H, Kurnia W and Tan P C 2008 Critical assessment and numerical comparison of electro-thermal models in EDM. J. Mater. Process. Technol. 203: 241-251

[5] Rebelo J C, Morao Dias A, Kremer D and Lebrun J L 1998 Influence of EDM pulse energy on the surface integrity of martensitic steels. J. Mater. Process. Technol. 84: 90-96

[6] Amorim F L and Weingaertner W L 2005 The influence of generator actuation mode and process parameters on the performance of finish EDM of a tool steel. J. Mater. Process Technol. 166: 411-416

[7] Snoeys R and Van Dijck F 1971 Investigation of electro discharge machining operations by means of thermo-mathematical model. CIRP Ann. 20: 35-36

[8] Erden A and Kaftanoglou B 1981 Thermo-mathematical modelling and optimization of energy pulse forms in Electric Discharge Machining (EDM). Int. J. Mach. Tool Des. Res. 21: 11-22

[9] DiBitonto D D, Eubank P T, Patel M R and Barrufet M A 1989 Theoretical models of the electrical discharge machining process: I. A simple cathode erosion model. $J$. Appl. Phys. 66: 4095-4103

[10] Pandey P C and Jilani S T 1986 Plasma channel growth and the resolidified layer in EDM. Precis. Eng. 8: 104-110

[11] Singh A and Ghosh A 1999 Thermo-electric model of material removal during electric discharge machining. Int. J. Mach. Tools Manuf. 39: 669-682

[12] Yadav V, Jain V K and Dixit P M 2002 Thermal stresses due to electrical discharge machining. Int. J. Mach. Tools Manuf. 42: $877-888$

[13] Salah N B, Ghanem F and Atig K B 2006 Numerical study of thermal aspects of electric discharge machining process. Int. J. Mach. Tools Manuf. 46: 908-911

[14] Dhanik S and Joshi S S 2005 Modeling of a single resistance capacitance pulse discharge in micro-electro discharge machining. J. Manuf. Sci. Eng. 112: 759-767 
[15] Kojima A, Natsu W and Kunieda M 2008 Spectroscopic measurement of arc plasma diameter in EDM. CIRP Ann. 57: 203-207

[16] Nagahanumaiah, Ramkumar J, Glumac N, Kapoor S G and DeVor R E 2009 Characterization of plasma in micro-EDM discharge using optical spectroscopy. J. Manuf. Process. 11: 82-87

[17] Han F, Cheng G, Fenf Z and Soichiro I 2009 Measurement of wire electrode temperature in WEDM. Int. J. Adv. Manuf. Technol. 41: 871-879

[18] Stephenson D A 1991 An inverse method of investigation deformation zone temperature in metal cutting. J. Eng. Ind. 113: 129-136

[19] Kim H J, Kim N K and Kwak J S 2006 Heat flux distribution model by sequential algorithm of inverse heat transfer determining workpiece temperature in creep feed grinding. Int. J. Mach. Tool Manuf. 46: 2086-2093

[20] Gostimirovic M, Kovac P and Sekulic M 2011 An inverse heat transfer problem for optimization of the thermal process in machining. Sadhana-Acad. Proc. Eng. Sci. 36: 489-504

[21] Kunieda M, Kowaguchi W and Takita T 1999 Reverse simulation of die-sinking EDM. CIRP Ann. 48: 115-118

[22] Saedodin S, Torabi M and Eskandar H 2010 Thermal analysis of workpiece under electrical discharge machining (EDM), using hyperbolic heat conduction model. Int. J. Adv. Des. Manuf. Technol. 3: 17-24
[23] Chiou W Y, Chen C I and Lu W S 2011 The inverse numerical solutions of the nonlinear heat transfer problem in electrical discharge machining. Numer. Heat Transfer A Appl. 59: 247-266

[24] Beck J V, Blackwell B and Clair C R 1985 Inverse Heat Conduction: Ill-Posed Problems. New York: WileyInterscience

[25] Alifanov O M 1994 Inverse Heat Transfer Problems. Berlin: Springer

[26] Deaconu A and Ciurea E 2013 Inverse feasibility problems of the inverse maximum flow problems. Sadhana-Acad. Proc. Eng Sci. 38: 199-209

[27] Tikhonov A N and Arsenin V Y 1977 Solution of Ill-Posed Problems. Washington: Winston \& Sons

[28] Ozisik M N and Orlande H R B 2000 Inverse Heat Transfer: Fundamentals and Applications. New York: Taylor \& Francis

[29] Gostimirovic M, Kovac P, Sekulic M and Skoric B 2012 Influence discharge energy on machining characteristics in EDM. J. Mech. Sci. Technol. 26: 173-179

[30] Jaeger J C 1942 Moving sources of heat and temperature at sliding contacts. J. Proc. R. Soc. N.S.W. 76: 203-224

[31] Brenner S and Scott R L 1994 The Mathematical Theory of Finite Element Methods. New York: Springer 